\title{
Sind die Extremitäten der Frösche regenerationsfähig?
}

\author{
Von \\ Dietrich Barfurth.
}

[Aus dem vergleichend-anatomischen Institut der kaiserlichen

Universität Jurjew Dorpat.]

Hierzu Tafel VI.

\section{Einleitung.}

Während die Urodelen verloren gegangene Gliedmaßen mit erstaunlicher Leichtigkeit und sogar oft im Überschuss regeneriren, ist diese Fähigkeit den Anuren ganz verloren gegangen oder doch sehr gering geworden. Freilich sind die Angaben der Forscher dariber außerordentlich widersprechend. Der hervorragendste Experimentator der älteren Zeit, SpallanzanI, erzielte positive Resultate: »Die jungen Frösche und Kröten thaten meiner Erwartung eine Genüge, indem sie neue Beine wieder bekamen « (4, pag. 65). Nachher fügt er hinzu, dass diese Reproduktion nicht so schnell wie beim Salamander erfolgt und »nicht allemal« (pag. 66).

Dagegen berichtet ein Experimentator der jüngeren Zeit, Frarsse, dass »seine Versuche über die Regenerationsfähigkeit der Extremitäten jüngerer und älterer Anuren ein durchaus negatives Resultat hatten « (3, pag. 35). Und an anderer Stelle: „Ein vollständig negatives Resultat erhielt ich dagegen bei den Larven unserer einheimischen Frösche und Kröten, auch wenn sie noch in sehr jugendlichem Alter standen. Stets wuchs auch unter den günstigsten Lebensbedingungen ein kleiner Conus an der verstümmelten Extremität nach, den ich jedoch niemals zur weiteren Ausbildung gelangen sah. Diese Thatsache ist um so auffälliger, als es ja bekannt ist, dass Frösche mit drei Hinterbeinen vorkommen, und solche zuerst wohl von J. van der Hokven und auch in verschiedenen populären Werken abgebildet sind. Mir selbst ist ein derartiges Exemplar leider niemals in die Hände gekommen und so kann ich mich auch 
über die Ursachen dieser Missbildung hier nicht weiter aussprechen * (3, pag. 103-104).

In gleichem Sinne äußert WeIsmanN: "Bekannt ist es auch, dass den Fröschen die abgeschnittenen Beine nicht wieder nachwachsen, auch nicht im Larvenzustand « (5, pag. 154).

Diese Angaben stehen also in schroffem Widerspruch mit den positiven Resultaten Spallanzani's, der als genialer Experimentator von unvergleichlichem Geschick neuerdings noch von PfLüger gepriesen wurde und dessen Angaben auch von mir so vielfach bestätigt werden konnten. Ich beschloss desshalb bei Gelegenheit einschlagiger Versuche auch diesen Gegenstand einer erneuten Prïfung zu unterziehen. Und da mir von früheren Experimenten über die Regenerationsfähigkeit der Chorda dorsalis (2) bei Urodelen bekannt war, wie wichtig es ist, bei solchen Versuchen gerade die jüngsten Stadien zu wählen, so nahm ich junge Froschlarven, bei denen eben erst die hinteren Extremitäten zum Vorschein kamen und weiterhin zwei etwas vorgeschrittene Stadien. Die Ergebnisse dieser Versuche waren mir recht interessant. Ich berichte jetzt zunächst über die Experimente.

\section{Eigene Versuche.}

1. Versuch. Am 22. Mai 1894 (neuen Stils) amputirte ich 20 kräftigen, im Institut gezogenen Larven von Rana fusea die rechte, eben erst zum Vorschein gekommene, hintere Extremität und zwei anderen die linke. Dieselbe ist noch so kurz, dass die Amputation etwas schwierig ist und nur mit einer kleinen scharfen Schere gut gelingt. Die abgeschnittenen Stummel wurden mit der Lupe kontrollirt; sie besaßen meistens drei, zum Theil fü $f$ stumpfe, kegelförmige Hervorragungen (Anlagen der Zehen). Nach der Operation fraßen die Thiere sofort am Fleisch eines abgestorbenen Axolotl. Sie wurden in einem mit Wasserpflanzen versehenen Gefäß gehalten und gut genährt. Öftere Besichtigung zeigte deutlich Regeneration der amputirten Extremität. Am 12. Juni ergab sich, dass noch 15 Thiere lebten, bei denen sämmtlich die abgeschnittene Extremität in Regeneration begriffen war. Bei vielen ist nicht nur der Oberschenkel, sondern auch der Unterschenkel mit einem nach außen umbiegenden schaufelförmigen Fortsatz, der Anlage des Fußes, regenerirt. Es handelt sich dabei nicht etwa um ein einfaches Auswachsen des centralen Amputationsstumpfes, sondern 
es liegt echte gestaltende Regeneration vor. Das ergiebt sich daraus, dass Ober- und Unterschenkel der regenerirten Extremität nur etwa halb so lang und viel diuner sind, als die der normalen Extremität; es folgt das ferner aus einem Vergleich mit den später zu erwähnenden Thieren eines anderen Versuches, die die Extremität nicht regenerirten. Es wurden drei Thiere in Mixtur (Alkohol + Glycerin + Wasser in einem früher [1] von mir angegebenen Verhältnis konservirt, die übrigen in ein anderes Gefäß gebracht. Dasselbe war mit Wasserpflanzen und Erde so bestellt, dass die Thiere nach Ausbildung der Extremitäten, wenn sie das Bestreben haben, aus dem Wasser zu gehen, sich niederlassen konnten. Das Gefäß wurde bedeckt. Die Entwickelung rerlief bei den meisten in etwas erhöhter Temperatur $\left(23^{\circ} \mathrm{C}\right.$.) recht günstig.

Am 19. Juni ergab sich folgender Befund:

Zwei Thiere sind todt, eins hat die abgesehnittene linke Extremität bis zur Anlage des Fußes, das andere den ganzen Fuß mit Zehenanlagen regenerirt. Diese beiden Thiere, sowie die gleich zu besprechenden Nr. 3-4 und Nr. 8-11 hatten die vorderen Extremitäten entwickelt und standen gerade vor oder in der Metamorphose.

Nr. 3 hatte die linke, Nr. 4 die rechte hintere Extremität vollständig regenerirt; am Fuß waren alle Zehen deutlich.

Nr. 4 hatte schon den Schwanz resorbirt.

Nr. 5-7 haben die vorderen Extremitäten noch nicht entfaltet, aber die amputirte h. E. in allen Theilen regenerirt; nur bei einem Thier ist der Fuß mit den Zehen etwas verkümmert.

Nr. 8-10 haben alle Extremitäten entwickelt, die hinteren Extremitäten vollständig regenerirt.

Nr. 11 hat die amputirte Extremität mit einem verkümmerten Fuß regenerirt, den Schwanz schon fast ganz resorbirt.

Nr. 12 ist ein ungewöhnlich großes kräftiges Thier (Fig. 4), welches die vorderen Extremitäten noch nicht ausgebildet hat, also in der Entwickelung an und für sich etwas hinter den anderen zuriick ist. Es hat trotzdem die amputirte Extremität vollständig regenerirt, nur sind alle regenerirten Theile etwas kürzer und schwächer.

Bei den regenerirten Extremitäten dieser sämmtlichen (und der gleich zu besprechenden) Versuchsthiere war der Oberschenkel am weitesten entwickelt und fast dem normalen der gegenseitigen Extremität gleich. Nach unten zu (distal) nimmt die Stärke der regenerirten Theile (Unterschenkel, Fuß) ab. 
2. Versuch. Am 23. Mai 1894 amputirte ich 12 Larven von Rana fusca die rechte hintere Extremität. Dieselbe war etwas weiter entwickelt, wie bei den Thieren des ersten Versuchs: die erste Anlage des Unterschenkels war zu sehen, wie es Fig. 12 (verglichen mit Fig. 1) demonstrirt. Auch diese Larven regenerirten sämmtlich mehr oder weniger vollständig und schnell die amputirte Extremität. Am 12. Juni lebten noch 9 Thiere, bei denen fast alle Stadien der Regeneration bis zur Anlage des Fußes vorlagen; an diesem Tage hatte noch keins alle vier Extremitäten. Es wurden wieder 3 Thiere konservirt und die ubrigen in derselben Weise, wie oben beschrieben, weiter gezüchtet.

Bei der Besichtigung am 17. Juni haben Nr. $1-4$ alle vier Extremitäten. Nr. 1 hat die r. h. E. ganz regenerirt, fast so lang wie die normale. Nr. 2 hat eine etwas verkümmerte r. h. E. regenerirt, an der ein Fuß mit zwei Fortsätzen (Zehenanlagen?) sichtbar ist. Nr. 3 und 4 haben die Extremität mit Fuß und allen Zehen regenerirt, nur sind alle Theile kürzer und schwächer, als normal.

Nr. 5 und 6 sind in der Entwickelung iberhaupt noch so weit zuriuck, dass die Vorderextremitäten noch gar nicht unter der Haut der Kiemenhöhle sichtbar sind und an der normalen (linken) hinteren Extremität der Oberschenkel mit dem Unterschenkel noch einen ganz stumpfen Winkel bildet. Trotzdem hat sich die amputirte r. h. E. unabhängig regenerirt: man sieht Ober- und Unterschenkel, Fuß und Zehen dentlich, nur wieder Alles etwas kürzer und dünner, als an der normalen Extremität. Es verläuft also die Regeneration auch hier bis zu einem gewissen Grade unabhängig von der direkten Entwickelung. Ganz dasselbe Resultat lieferten mir die frïheren Versuche über Regeneration der Schwanzspitze bei Froschlarven: die Schwanzspitze wurde regenerirt, mochten die Extremitäten schneller oder langsamer sich entwickeln (1).

3. Versuch. Am 24. Mai amputirte ich 11 Larven von Rana fusca die rechte, and 2 Larven die linke hintere Extremität. Dieselben waren schon ziemlich lang: der Oberschenkel bildete mit dem Unterschenkel einen stumpfen Winkel (vgl. Fig. 22).

Bei der genaueren Besichtigung am 12. Juni fanden sich noch neun Thiere lebend vor. Von diesen hatten drei die vorderen Extremitäten entwickelt und die Schwanzspitze zeigte eben die für die beginnende Metamorphose charakteristische Pigmentirung und Schrumpfung. Von diesen hat nur ein Thier (Fig. 24) Oberschenkel, 
Unterschenkel und einen verktimmerten $\mathrm{Fuß}$ regenerirt. Die beiden anderen Thiere zeigen an der Amputationsstelle der r. h. E. einen kurzen kegelförmigen Stummel mit dunkelm Centrum: von einer Regeneration keine Spur!

Bei vier anderen Thieren ist die $1 . \mathrm{h}$. E. fast vollständig entwickelt: der Oberschenkel hat die fur die Reife charakteristische Adduktion an den Leib, der Unterschenkel bildet mit dem Oberschenkel einen spitzen Winkel. Die rechte hintere Extremität ist nicht regenerirt! Der Amputationsstumpf ist kegelförmig, wie oben beschrieben. Ein einziges von den Thieren, bei denen die $l$. h. E. amputirt worden war, und zwar das jüngste von den noch lebenden, hat die 1. h. E. in Gestalt eines schlanken kegelförmigen Fortsatzes von ca. $2 \mathrm{~mm}$ Länge zu regeneriren begonnen! Es wurden die drei zuerst beschriebenen Larven konservirt und die übrigen in oben angegebener Weise weiter gezüchtet.

Am 19. Juni leben noch drei Thiere. Nr. 1 und 2 haben die vorderen Extremitäten entwickelt, aber die r. h. E. ist bei Nr. 1 gar nicht, bei Nr. 2 nur als kurzer Stummel regenerirt! $\mathrm{Nr}, 3$ hat die normale 1. h. E. fast ganz entwickelt, aber ihr Widerpart hat sich gar nicht regenerirt.

\section{Besprechung der Ergebnisse.}

Die Temperatur wurde bei diesen Versuchen im Einzelnen nicht weiter beriicksichtigt, weil es unnöthig war; sie betrug Anfangs ca. $20^{\circ} \mathrm{C}$, später ca. $23^{\circ} \mathrm{C}$. Kleine Unterschiede der Temperatur in den einzelnen Versuchsgefäßen waren ohne Zweifel vorhanden, sind aber fuir das Gesammtergebnis ohne Bedeutung.

Das Gesammtergebnis ist eine glänzende Rechtfertigung Spallaxzani's: die Extremitäten der jungen Frösche, d. h. der Froschlarven, sind in der That regenerationsfähig!

Aber auch für die abweichenden Angaben FraIsse's geben meine Versuche wahrscheinlich eine Erklärung. Ich habe gefunden; dass sehr junge Stadien der sich entwickelnden Extremitäten durchaus regenerationsfähig sind (Versuch 1 und 2), dass aber schon bei etwas entwickelteren Larven die Regenerationsfähigkeit fast a uf Null reducirt ist! (Versuch 3.) Da Fraisse über das Entwickelungsstadium der von ihm amputirten Extremitäten keine näheren Angaben gemacht hat, haite ich mich zu der oben gegebenen Erklärung für berechtigt. 
Ganz allgemein lehren meine Versuche, dass positive Resultate bei solehen Experimenten niemals durch negative umgestoßen werden können. Und das würde auch gelten, wenn etwa ein späterer Experimentator den Nachweis führen könnte, dass meine positiven Resultate nur. ftir die Dorpater Frösche gelten!

Ergebnisse.

1) Die Extremitäten der Larven von Rana fusca sind regenerationsfähig; damit wird eine alte Angabe Spallanzany's gegenüber FraIsse bestätigt.

2) Die Regenerationsfähigkeit dieser Extremitäten nimmt mit fortschreitender Entwickelung sehr schnell ab.

3) Die Regeneration ist bis zu einem gewissen Grade unabhängig von der Entwickelung.

\section{Litteraturverzeichnis.}

1) Barfurth, Dietrich, Versuche über die Verwandlung der Froschlarven. Archiv f. mikr. Anatomie, 29. Bd.

2) Derselbe, Zur Regeneration der Gewebe. Archiv f. mikr. Anatomie 1590.

3) Fraisse, P., Die Regeneration von Geweben und Organen bei den Wirbelthieren etc. Cassel und Berlin, 1885.

4) Spalcanzani, Physikalische und mathematische Abhandlungen. Mit Kupfern. Leipzig, 1769.

5) Weismann, A., Das Keimplasma. Eine Theorie der Vererbung. Jena, 1892.

\section{Erklärung der Abbildungen.}

Tafel VI.

Die Versuchsthiere sind in natürlicher Größe unter Kontrolle mit der Lupe gezeichnet. Die Stellung der Extremitäten entspricht meistens der Norm, bei einigen ist ohne Weiteres ersichtlich, dass sie bei Eintritt des Todes in der Konservirungsfliissigkeit etwas abnorm gestreckt wurden. Das Stadinm der Extremitätenansbildung bei den Thieren der drei verschiedenen Versuche ist durch ein Probeexemplar (1, 12, 22) veranschanlicht. Zur besseren Demonstration der Extremititen sind alle Thiere von der Ba uchseite gezeichnet.

\section{Versuch I.}

Nr. 1 zeigt die hinteren Extremitäten als kurze Stummelchen, wie sie bei Versuch I, Nr. 2-11, am Tage der Amputation beschaffen waren.

Nr. 2-3 zeigt den Erfolg der Regeneration nach 21 Tagen. Ober- und Unterschenkel sind regenerirt, aber kiirzer und schwächer als normal, auch die Fußanlage, bei Nr. 3 sogar drei Zehenanlagen, sind schon vorhanden. 
Nr. 4-11 zeigt die Regeneration bei Versuch I nach 28 Tagen.

Nr. 4-6 haben die hinteren Extremitäten fast vollständig entwickelt, die amputirte rechte Extremität ist überall regenerirt, bei dem ungewöhnlich kräftigen Thier Nr. 4 unter voller Ausbildung der Zehen, bei Nr. 6 am schwächsten unter abnormer Adduktion des Fußes.

Nr. 7 hat die l. v. Extremität schon entwickelt, die rechte sitzt noch unter der Haut der Kiemenhöhle (gewöhnlich bricht die rechte Extremität zuerst durch!). R. h. Extremität mit vier Zehen regenerirt.

Nr. 8 hat alle Extremitäten entwickelt, die ampatirte r. h. E. mit vier vollständigen Zehen und einer höckerförmigen Anlage der fünften regenerirt.

Nr. 9-11 haben den Schwanz theilweise oder ganz resorbirt, alle Extremitäten entwickelt und die amputirte regenerirt; bei Nr. 9 sind nur vier Zehen vorhanden, bei Nr. 11 ist der Fuß verkümmert, abwärts (also bei Bauchlage aufwïrts) verkriimmt.

\section{Versuch II.}

Nr. 12 Probethier, zeigt die Entwickelung der h. Extremitäten bei Anstellnng des Versuches (Nr. 13-21); die erste Anlage der unteren Extremität ist durch eine leise Verschmächtignng und Krümmung angedentet.

Nr. 13-15 zeigt drei Thiere, bei denen 20 Tage nach dem Versuch die Entwickelnng und Regeneration am weitesten vorgeschritten war. Bei Nr. 13 und Nr. 14 Oberschenkel and Unterschenkel, vom Fuß keine Spur, Nr. 15 hat auch einen Fuß mit drei Zehen regenerirt; bei 14 und 15 schimmern die vorderen Extremitäten durch die Haut der Kiemenhöhle durch, sind aber noch nicht zum Durchbruch reif.

Nr. 16-21 wurden 27 Tage nach der Amputation konservirt.

Nr. 16 hat den Ober- und Unterschenkel regenerirt, den Fuß nicht, Nr. 17 die ganze Extremität mit Fuß, was hier auffallig ist, weil die linke nicht amputirte Extremität noch nicht die Zeichen vollständiger Entwickelung (rechtwinklige oder spitzwinklige Gelenkstellung an Ober- und Unterschenkel aufweist. Hier hat also die Regeneration die Entwickelung fast ganz eingeholt, was für die Selbständigkeit der ersteren spricht.

Nr. 18-21 zeigen Resorption des Schwanzes, Ausbildung aller Extremititen Regeneration der amputirten Extremität.

Nr. 18 hat einen verkïmmerten zweizehigen Fuß regenerirt; Spuren ron anderen noch zu regenerirenden Zehen waren nicht vorbanden.

Nr. 19-21 haben den Fuß mit 5 Zehen in normaler Stellung regenerirt.

\section{Versuch III.}

Nr. 22 Probethier, zeigt die Entwickelung der Extremität bei Anstellung des Versuches (Nr. 23-27; der FuB mit allen Zehen war ausgebildet.

Nr. 23-25 zeigt drei Thiere, die 19 Tage nach der Amputation konservirt wurden. Nur Nr. 24 hat Oberschenkel, Unterschenkel und einen verkümmerten FuB regenerirt. Die anderen beiden Thiere, ebenso die 26 Tage nach der Amputation konservirten $\mathrm{Nr} .26-2 \tau$ zeigen keine Spur einer Regeneration. 